\title{
Efflux Pump AdeABC Assessment in Acinetobacter baumannii Strains Isolated in a Teaching Hospital
}

Claudia Rizzo, Nadia Marascio*, Emilia Zicca, Grazia Pavia, Angela Quirino, Angelo Giuseppe Lamberti, Maria Carla Liberto and Alfredo Focà

Department of Health Sciences, Institute of Microbiology, School of Medicine, University of Magna Graecia, Viale Europa, Germaneto, 88100 Catanzaro, Italy

"Corresponding author: Nadia Marascio, Department of Health Sciences, Institute of Microbiology, School of Medicine, University of Magna Graecia, Viale Europa, Germaneto, 88100 Catanzaro, Italy, Tel: 3909613694457; Fax: 3909613697760; E-mail: nadiamarascio@gmail.com

Rec Date: Jun 13, 2016; Acc Date: Jul 21, 2016; Pub Date: Jul 29, 2016

Copyright: $\odot 2016$ Rizzo C, et al. This is an open-access article distributed under the terms of the Creative Commons Attribution License, which permits unrestricted use, distribution, and reproduction in any medium, provided the original author and source are credited.

\begin{abstract}
Over the past twenty years the worldwide clinically impact of Acinetobacter baumannii (A. baumannii) demonstrated its etiopathogenetic relevance. During a previously retrospective study in a teaching hospital, between January 2011 and February 2015, we observed increasingly infections caused by $A$. baumannii associated with antibiotic multi-resistance. Tigecycline, the first member of the glycylcycline class, is an effective option for the treatment of such infections even if, due to its increased clinical use, tigecycline resistant isolates have recently emerged. In $A$. baumannii several mechanisms are associated with a tigecycline decrease susceptibility, among these, expression efflux pump AdeABC and the presence of insertion sequence (IS) in the adeRS operon. About that, we decided to analyze adeB and ades genes in 24 MDR $A$. baumannii clinical isolates, selected on the different tigecycline phenotype. The study of $a d e B$ and adeS genes was performed by an in-house polymerase chain reaction (PCR) and by Sanger sequencing method. According to literature ade $B$ and adeS genes were detected in all MDR A. baumannii isolates tested. Therefore our attention has focused on two resistant tigecycline clinical strains $(\mathrm{ACl} 2313$ and $\mathrm{ACl}$ 1213), with a MIC value >8. In particular the $\mathrm{ACl} 2313$ strains, showed the presence of an IS in the adeS gene. Then, adeS sequence analysis identified ISAba1 insertion. Moreover, adeB gene expression was evaluated by an in-house SYBR Green l-based real-time RT-PCR. We found an over expression of ade $B$ gene in $A C l 2313$ strain, according to IS presence on adeS gene, while the lack of ade $B$ overexpression in $\mathrm{ACl} 1213$, still resistant to tigecycline, could be due to different resistance mechanisms.
\end{abstract}

Keywords: Tigecycline; A. baumannii isolates; Glycylcycline; Antibiotic resistance

\section{Introduction}

Acinetobacter baumannii (A. baumannii) is an opportunistic pathogen that commonly causes nosocomial infections, as pneumonia, bloodstream and urinary tract infections, particularly in the intensive care unit [1]. Multi drug resistant (MDR) A. baumannii isolates have been reported worldwide and their increasing prevalence has led to limited therapeutic choice [2].

Tigecycline, the first member of the glycylcycline class of antibacterial agents, remain effective option for the treatment of these infections. However, due to its increased clinical use, tigecycline resistance is recently emerging [3].

Several studies have indicated that tigecycline resistance of $A$. baumannii is associated with the over expression of AdeABC efflux system [4,5]. A two component system containing adeS and AdeR, a sensor kinase and a response regulator respectively, are responsible for modulating AdeABC efflux pump [6]. Moreover, nucleotide/amino acid variations as well as the presence of insertion sequences (IS), such as ISAba1, in the adeRS operon have been related to the over expression of the adeABC efflux pump, decreasing $A$. baumannii susceptibility to tigecycline [7]. However, the exact mechanisms of resistance and the relationship between the level of expression of efflux pumps and the minimal inhibitory concentration (MIC, mg/liter) of tigecycline have not yet been clearly elucidated. Also, whether clinical isolates with resistance to tigecycline, originating from the same geographic locations, possess similar mechanisms of resistance is still unclear.

During a retrospective study in a teaching hospital, between January 2011 and February 2015, we evaluated distribution and antibiotic resistance of $A$. baumannii strains isolated from patients admitted to four hospital units (medical units, surgical units, cardiac intensive care unit and the intensive care unit). A. baumannii isolates were collected from several sites such as blood culture, bronchial aspirate, bronchoalveolar lavage, central venous catheter, urine, and bladder catheter tip. Data collected showed an increasingly infections caused by $A$. baumannii associated with antibiotic multi-resistance (unpublished data). In particular on 83 strains, isolated in the last year, the percentages of MDR and pan drug resistant (PDR) A. baumannii were $75 \%$ and $13 \%$ respectively.

\section{Objective}

Since the observed high frequency of multi drug resistant $A$. baumannii in our hospital, the aim of this study was to assess efflux pump AdeABC in $24 \mathrm{MDR} A$. baumannii strains, selected on the different tigecycline phenotype.

\section{Study Design}

Twenty-four clinical isolates of A. baumannii, collected at "Mater Domini" University Hospital of Catanzaro, Southern Italy, from January 2013 to February 2015, were selected according to tigecycline phenotype $(0.5 \leq \mathrm{MIC} \geq 8)$. Isolates were identified by using VITEK 2 system (bioMérieux) and by mass spectrometry MALDI-TOF MS 
(bioMèrieux, France). A. baumannii ATCC 19606, an environmental A. baumannii strain, previously isolated from Mediterranean Sea water sample, and $A$. haemolyticus clinical isolate were also included in our study, as controls. For MALDI-TOF MS identification, bacterial cells from blood agar culture were processed according to the manufacturer's instructions. MALDI-TOF peaks were compared with reference spectra using SARAMIS integrated database. Antimicrobial susceptibility to tigecycline was determined by VITEK 2 system (ASTN201/AST-N203 cards) and United States Food and Drug Administration (FDA) breakpoint criteria for Enterobacteriaceae [8], to perform gene sequencing, bacterial DNA was extracted using UltraCleanTM Microbial DNA-MoBio Kit. The adeB and adeS genes were amplified by an in-house PCR and following primers: adeB Forward (5'-TAT CCT GGT GCT ACA GCT AA-3') and adeB Reverse (5'-CTC GA GAC TAG ACA A TTG CC-3'), ades Forward (5' GTA GAC TGG ATC TGG TTA GC-3') and adeS Reverse (5'-AGT GGA CGT TAG GTC AAG TT-3'). PCR products of adeB gene, from ACI 4614, ACI 2313, ACI 1213, and ATCC 19606 isolates, as well as adeS gene, from ACI 2313 and ATCC 19606 strains, were sequenced. Following amplicon purification, using PCR Illustra MicroSpin S-300 Colonne HR (Gelifesciences UK), Sanger sequencing was performed by ABI PRISM 3500 Genetic Analyzer (Applied BiosystemsTM, life technologies). Sequences were analyzed with BLASTN program of the NCBI Basic Local Alignment Search Tool (BLAST, https:// blast.ncbi.nlm.nih.gov/).

The ACI 4614, ACI 2313, ACI 1213, and ATCC 19606 isolates were also evaluated for adeB gene expression using an in-house developed SYBR Green I-based real-time RT-PCR. Total RNA was isolated by Trizol $^{\circledR}$ Reagent (AmbionTM, life technologies). The High Capacity cDNA Reverse Transcription Kit (Applied BiosystemsTM) for cDNA synthesis was used. Real-Time PCR was run in a LightCycler Instrument (Roche Molecular Biochemicals, Indianapolis, IN), using same primers described above. Of each cDNA, housekeeping gene and target gene in triplicate were determined. The amplicon specificity was confirmed by melting curve analysis, previously established (adeB melting temperature $85.28^{\circ} \mathrm{C} \pm 0.5^{\circ} \mathrm{C}$ ). Expression level of housekeeping gene was used to normalize the abundance of the tested transcripts. Comparative threshold cycle (CT) was used to determine transcript fold changes present in ACI 4614, ACI 2313 and ACI 1213 compared to ATCC 19606 [9].

\section{Results}

The identification analyses of 24 strains were confirmed by two methods. At first, isolates were identified by VITEK 2 system. Since Acinetobacter calcoaceticus-baumannii (ACB complex) are phenotypically indistinguishable when evaluated by biochemical characteristics, the clinical isolates were also identified by mass spectrometry MALDI-TOF MS. Mass spectrometry MALDI-TOF MS identified A. baumannii specie with high reliability, founding $\mathrm{m} / \mathrm{z}$ $5747 / 5749$ range peaks [10]. The VITEK 2 system was also used to determine antibiotic susceptibilities. The MIC $\geq 8$, related to tigecycline resistance, was found in ACI 1213 and ACI 2313 strains, while, the others isolates showed a MIC value ranging 0.5-4.

Following in-house PCR, in all MDR $A$. baumannii clinical isolates as well as in $A$. baumannii ATCC 19606 reference strain, ade $B$ (Figure 1) and adeS (data not shown) were found. Conversely, as expected, both environmental $A$. baumannii strain and $A$. haemolyticus clinical isolates were lacking in these genes. The ade $B$ Sanger sequencing from ACI 4614, ACI 1213, ACI 2313 and ATCC 19606 isolates was performed. Sequencing analysis showed $99 \%$ homology with the adeB sequence of $A$. baumannii strains included in NCBI-BLAST. Additionally, in ACI 2313 isolate, ade $B$ showed a threefold higher relative expression (Figure $2 \mathrm{~A}$ and $2 \mathrm{~B}$ ). Moreover, we sequenced adeS gene from the same isolate (ACI 2313), showing a singular electrophoretic migration pattern, and ATCC 19606 strain, as control. Sequencing analysis demonstrated that ACI 2313 tigecycline resistant isolate carried ISAbal insertion sequence.

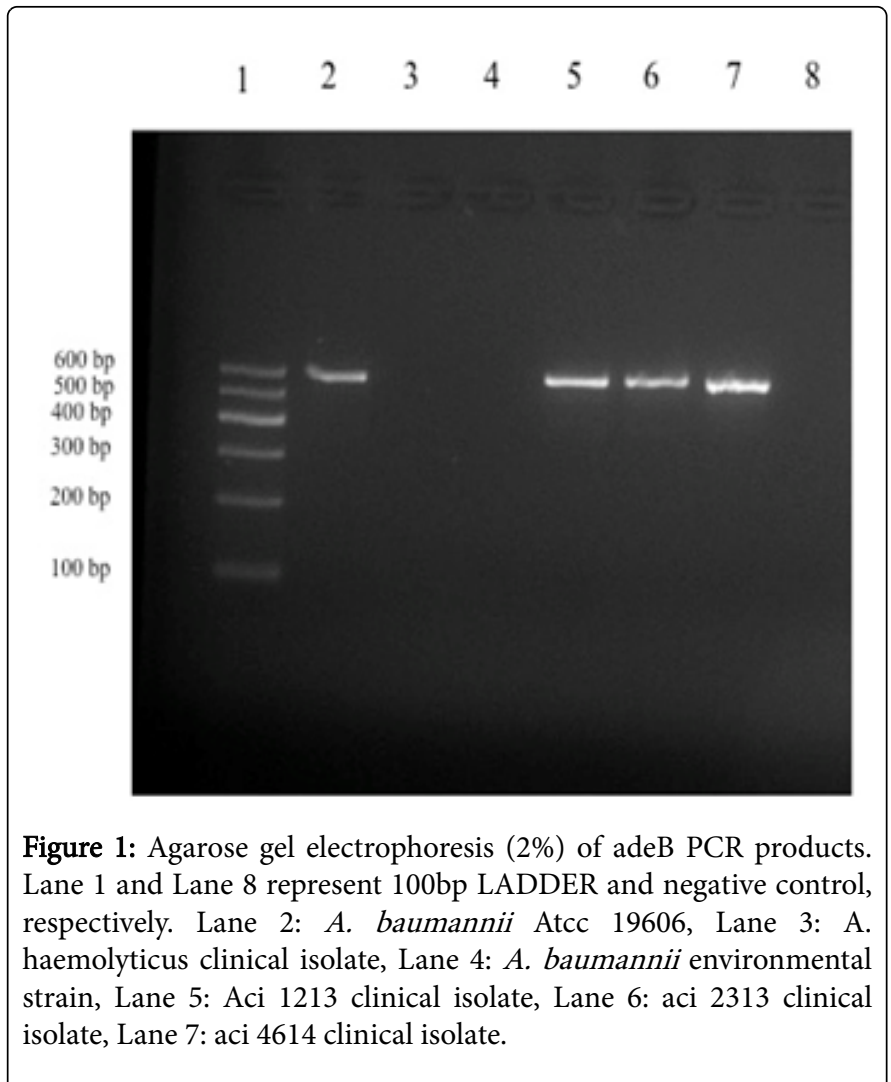

\section{Discussion}

Different studies have reported the role of $A$. baumannii efflux pumps in resistance to clinically relevant antibiotics [11]. AdeABC efflux pump has been well characterized, it is apparently not well expressed in wild-type strains [12], and contributes significantly to acquire multi drug resistance in worldwide clinical isolates, including resistance to tigecycline increasingly reported since 2007 [13-17]. However tigecycline is one of the few remaining therapeutic options for treating infections caused by MDR A. baumannii. Previous report [4] suggested that decreased susceptibility to tigecycline in the complex Acinetobacter calcoaceticus-Acinetobacter baumannii is associated with overexpression of efflux pump AdeABC.

Recently, the overexpression of AdeABC was referred as the prevalent mechanism in tigecycline resistant $A$. baumannii clinical isolates, and a linear relationship was found between ade $B$ gene expression levels and tigecycline MICs [17]. Our data showed that, even if adeB were detected in all MDR A. baumannii strains tested, differences in $a d e B$ gene expression have been found. 


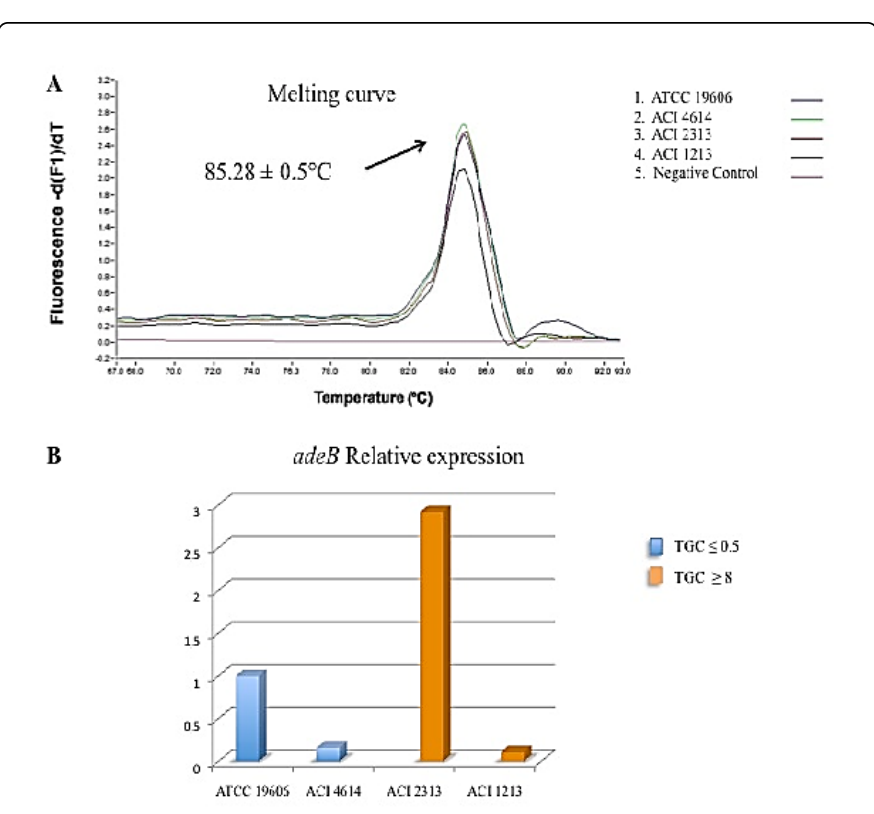

Figure 2: adeB transcript analysis by real time RT PCR assay. Melting curve analysis (A) of ACI 4614, ACI 2313, ACI 1213 and ATCC 19606. adeB relative gene expression (B) in ACI 4614, ACI 2313, ACI 1213 clinical isolates compared with A. baumannii ATCC 19606. Tigecycline (TGC) MIC is in mg/liter.

Indeed, in the 2 clinical isolates, sharing a MIC value $>8$, we determined substantial differences; in particular, in just ACI 2313 isolate, adeB showed a higher relative expression.

The AdeABC efflux pump is regulated by a two component system, AdeS sensor kinase and AdeR response regulator, encoded by the adeRS operon. It has been reported that overexpression of the AdeABC system is due to mutation in adeRS operon, included the presence of insertional sequence, such as ISAba1, one of the most frequent IS found in clinical isolates [6-7]. When we detected and analyzed adeS in our clinical isolates, ACI 2313 strain showed a distinct electrophoretic migration pattern and its partial sequencing matched to ISAba1. The analysis to cover the internal gap (around $400 \mathrm{bp}$ ) and to obtain the entire IS is still in progress.

In conclusion, further examination on additional tigecycline resistant $A$. baumannii clinical isolates, spreading in our area, are required. Mechanisms leading to tigecycline resistance, in particular in strains originating from the same geographic locations, will contribute to check the future prevalence of this resistance and to understand the A. baumannii pan drug resistance evolution.

\section{Conflicts of interests}

The authors declare that they have no competing financial interests.

\section{References}

1. Peleg AY, Seifert H, Paterson DL (2008) Acinetobacter baumannii: emergence of a successful pathogen. Clin Microbiol Rev 21: 538-582.

2. Gordon NC, Wareham DW (2009) A review of clinical and microbiological outcomes following treatment of infections involving multidrug-resistant Acinetobacter baumannii with tigecycline. J Antimicrob Chemother 63: 775-780.

3. Sun Y, Cai Y, Liu X, Bai N, Liang B, et al. (2013) The emergence of clinical resistance to tigecycline. Int J Antimicrob Agents 41:110-116.

4. Ruzin A, Keeney D, Bradford PA (2007) AdeABC multidrug efflux pump is associated with decreased susceptibility to tigecycline in Acinetobacter calcoaceticus-Acinetobacter baumannii complex. J Antimicrob Chemother 59: 1001-1004.

5. Yoon EJ, Courvalin, P, Grillot-Courvalin C (2013) RND-type efflux pumps in multidrug-resistant clinical isolates of Acinetobacter baumannii: major role for AdeAB overexpression and AdeRS mutations. Antimicrob Agents Chemother 57: 2989-2995.

6. Li XZ, Plésiat P, Nikaido H (2015) The Challenge of Efflux-Mediated Antibiotic Resistance in Gram- Negative Bacteria. Clin Microbiol Rev 28 : 337-418.

7. Sun JR, Perng CL, Chan MC, Morita Y, Lin JC, et al. (2012) A truncated AdeS kinase protein generated by ISAbal insertion correlates with tigecycline resistance in Acinetobacter baumannii. PLoS One 7: e49534.

8. Jones RN, Ferraro MJ, Reller LB, Schreckenberger PC, Swenson JM, et al. (2007) Multicenter studies of tigecycline disk diffusion susceptibility results for Acinetobacter spp. J Clin Microbiol 45: 227-230.

9. Pfaffl MW (2001) A new mathematical model for relative quantification in real-time RT-PCR. Nucl Acids Res 29: e45.

10. Sousaa C, Botelhoa J, Silvaa L, Grossoa F, Nemecb A, et al. (2014) MALDI-TOF MS and chemometric based identification of the Acinetobacter calcoaceticus-Acinetobacter baumannii complex species. Int J Med Microbiol 304: 669-677.

11. Coyne S, Courvalin P, Perichon B (2011) Efflux-mediated antibiotic resistance in Acinetobacter spp. Antimicrob Agents Chemother 55:947953.

12. Magnet S, Courvalin P, Lambert T (2001) Resistance-nodulation cell division-type efflux pump involved in aminoglycoside resistance in Acinetobacter baumannii strain BM4454. Antimicrob Agents Chemother 45: 3375-3380.

13. Sun Y, Cai Y, Liu X, Bai N, Liang B, et al. (2013) The emergence of clinical resistance to tigecycline. Int J Antimicrob Agents 41: 110-116.

14. Hornsey M, Ellington MJ, Doumith M, Thomas CP, Gordon NC, et al. (2010) AdeABC-mediated efflux and tigecycline MICs for epidemic clones of Acinetobacter baumannii. J Antimicrob Chemother 65: 1589-1593.

15. Fernando D, Zhanel G, Kumar A (2013) Antibiotic resistance and expression of resistance-nodulation-division pump- and outer membrane porin-encoding genes in Acinetobacter species isolated from Canadian hospitals. Can J Infect Dis Med Microbiol 24: 17-21.

16. Deng M, Zhu MH, Li JJ, Bi S, Sheng ZK, et al. (2014) Molecular epidemiology and mechanisms of tigecycline resistance in clinical isolates of Acinetobacter baumannii from a Chinese university hospital. Antimicrob Agents Chemother 58: 297-303.

17. Yuhan Y, Ziyun Y, Yongbo Z, Fuqiang L, Qinghua Z (2016) Over expression of AdeABC and AcrABTolC efflux systems confers tigecycline resistance in clinical isolates of Acinetobacter baumannii and Klebsiella pneumoniae. Rev Soc Bras Med Trop 49: 165-171. 